九州大学学術情報リポジトリ

Kyushu University Institutional Repository

\title{
Beiträge Zur Biologie Der Pantopoden
}

Arita, Keizo

Zoologisches Laboratorium, Kaiserliche Kyushu-Universitat

https://doi.org/10.5109/22585

出版情報 : 九州大学大学院農学研究院紀要. 5(6)，pp.271-288，1937-03. Kyushu Imperial University バージョン：

権利関係 : 
Journal of the Department of Agriculture, Kyûshû Imperial University, Vol. 5, No. 6 , May 25, 1937

\title{
BEITRÄGE ZUR BIOLOGIE DER PANTOPODEN
}

\author{
Keizô ARITA
}

\section{EINLEITUNG}

Die Pycnogoniden oder Pantopoden, wie man sie in neuer Zeit meist nennt, um eine Verwechselung mit der Familie Pycnogonidae $z u$ vermeiden, sind eine jener kleinen distinkten Formengruppen, die, von den grossen Arthropoden-Klassen in vielen Beziehungen abweichend, eine merkwürdige Stellung innerhalb derselben einnehmen. In der Regel sind solche isolierte Gruppen wegen des Interessanten, das ihre systematische Stellung bietet, am eingehendsten untersucht worden und am besten bekannt; bei den Pantopoden scheint es indessen nur bis $z u$ einem gewissen Grade der Fall zu sein. Während die Systematik innerhalb der Gruppe schon einigermassen klar gelegt ist, herrschen über ihre Beziehung $z \mathrm{u}$ den anderen Klassen noch verschiedene Ansichten. Am ungenügendsten sind wir über die Biologie der Pantopoden unterrichtet. Zusammenhängende Untersuchungen darüber sind nur von wenigen Autoren angestellt worden, von denen es neben Cole, Loman und PRELL, waren, die bei ihrer ausführlichen Beschäftigung mit der Biologie der Pantopoden auf Grund von Beobachtungen an lebendem Material wichtige Beiträge geliefert haben. Ausserdem finden sich noch in einigen systematisch-anatomischen Arbeiten verstreute kurze Notizen über die Lebensweise der beschriebenen Arten. Allerdings handelte es sich bei den untersuchten Tieren gewöhnlich um die leicht lebend $\mathrm{zu}$ erhaltenen Küstenformen. Über die grossen, freilebenden Arten der Tiefsee scheint dagegen in biologischer Hinsicht so gut wie nichts bekannt zu sein.

i) Arbeiten aus dem Zoologischen Laboratorium der Kaiserlichen Kyûshû Universität zu Fukuoka, Nr. 94. 
Als ich dann im Frühling des Jahres 1932 an Herrn Professor Dr. Hiroshi Ohshima mit der Bitte herantrat, unter seiner Leitung über Zoologie arbeiten zu dürfen, kam er meinen Wünschen mit der grössten Liebenswürdigkeit entgegen, und rief in mir grosses Interesse für eine biologische Bcobachtung der Pantopoden wach. Gleichzeitig sah ich, welch reiches Material für diesbezügliche Untersuchungen in der nächsten Umgebung zu erlangen war. Es ist mir eine ehrende Pflicht, meinem hochverehrten Lehrer, Herrn Professor OHshima, an dieser Stelle für das reiche Interesse, das er meiner Arbeit schenkte, und für die mannigfaltigen Anregungen, durch welche er sie förderte, meinen herzlichsten Dank auszusprechen. Gleichzeitig möchte ich auch Herrn Professor Dr. Nisuke TAKAHASH für die freundlichen Anleitungen bei der künstlichen Züchtung, durch welche meine Lntersuchungen wesentlich erleichtert wurden, meinen verbindlichen Dank aussprechen. Ferner bin ich meinem Freunde Herrn Dr. Kan-ichi TASHIRo für die wohlwollende Hilfe bei dieser Arbeit und Herrn Kô-ichirô Yasumoto für die bereitwillige Unterstützung beim Beschaffen des Materials zu Dank verpflichtet.

Die Morphologie von Nymphonella tapetis OHSHIMA, welche eine in Paphia (Tapes) philippinarum und Protothaca jedoensis parasitierende Pantopode ist, wurde bereits von Herrn Professor OHSHIMA $(1927 \mathrm{a}, \mathrm{b}, 1933,1935)$ so eingehend beobachtet und beschrieben, dass sich kaum etwas hinzufügen lässt, jedoch weist die Biologie noch ungelöste Fragen auf. Daher sind die im folgenden mitgeteilten Ergebnisse fast ausschliesslich biologischer, weniger morphologischer Natur; wenn darin auslaufende Fragen soweit als irgend möglich ausgeschaltet wurden, z. B. Nerven-, und Blutgefässsystem u.a., so geschah es in der Hoffnung, dass mir später Gelegenheit zur Ausfüllung dieser Lücken gegeben sein werde.

\section{HISTORISCHES}

LOMAN schreibt: „An Pycnogoniden sind noch wenig biolo. gische Studien gemacht. In der ausführlichen Monographie von DoHRN über die Pantopoden des Golfes von Neapel, 1881, findet man bei aufmerksamem Lesen viele Bemerkungen, die der Verfasser im Verlaufe seiner Untersuchungen zu machen Gelegenheit fand. Da sie aber in die anatomische Auseinandersetzung eingeflochten sind, bilden sie kein zusammenhängendes Ganzes und geben dem Leser 
nur ein unvollkommenes Bild von der Lebensweise dieser Tiere. Der erste, der uns näher bekannt macht mit der Biologie einer amerikanischen Art, ist CoLE, dem wir manche wichtige Mitteilung verdanken. Zuerst gibt er uns eine genaue Beschreibung von der Weise, wie sich diese Tiere fortbewegen, sei es kriechend oder schwimmend, dann bespricht er den Einfluss des Lichtes, und kommt zum selben Ergebnis, wie LoEB, der zeigte, dass sie positiv heliotropisch sind. Und endlich hat er wahrgenommen, wie das Männchen die Eier des Weibchens übernimmt, und er gibt uns eine deutliche Beschreibung dieser anscheinend einfachen Erscheinung, die bereits früher, obgleich unvollständig, von HoEk wahrgenommen worden war."

Loman (1907), der seine Feststellung an Phoxichilidium femoratum (RATHKE) machte, studierte zwar auch die Bewegungen aber interessierte sich vor allem für die Nahrungsaufnahme, die Atmung, die Fortpflanzung und die Entwicklung mit allen Nebenerscheinungen. Die wichtigen Mitteilungen über die Lebensweise brachte sodann PRELL (1910), der an den verschiedenen Pantopoden-Formen die Nahrungsaufnahme, die Bewegungen u. a. untersuchte, letztere besonders beim Schwimmen, und dabei auch ein Schema für die Phasenfolge solcher Bewegungen aufstellte. Bei fast allen genannten Arbeiten wurden die Ausführungen der Autoren durch viele und gute Abbildungen erläutert

\section{MATERIAL UND METHODE}

Meine Untersuchungen sind alle an Nymphonella tapetis angestellt worden, von denen man leicht Hunderte sammeln und in Aquarien mit sich sclbstreinigendem Wasser am Leben erhalten kann. Unter dem Mikroskop wurden sie in das flache Glasschälchen gebracht (von der in der Bakteriologie gebrauchten Form), sodass Vergrösserungen bis ungefähr $200 \cdot$ fach noch möglich waren ${ }^{1)}$. Durch künstliche Züchtung der Larven, welche sorgfältig aus ihrem Wirte herausgenommen wurden, gelang es mir, geschlechtsreife Tiere $z u$ bekommen, und ich studierte die Biologie und ein wenig die Entwicklungsgeschichte.

1) Um sie für genauere Untersuchungen ruhig $\mathrm{zu}$. halten, erwies es sich als praktisch, eine dünne Glasplatte direkt auf das Tier zu legen, sodass es am hohlgeschliffenen Objektträger liegt; in dieser Lage kann man Nymphonella stundenlang ohne Schaden unter dem Mikroskop beobachten. 
DOHRN (1881) erzählt von einem Weibchen seiner Barana castelli (= Aschorhynchus c.), das er quer durchschnitt, um den vorderen Teil anatomisch $\mathrm{zu}$ untersuchen, wobei der hintere mit den Extremitäten V bis VII, noch vier Wochen lebend blieb ${ }^{13}$. Die Pantopoden wird man im Aquarium mit fliessendem Seewasser leicht am Leben halten können; auch LOEB (1896), COLE (1901), LOMAN (1907) und PRELI. (1910) hielten das Tier etwa wochenlang am Leben in Schalen entweder mit Wasser und Sand am Boden, oder nur mit Wasser und sorgten nur für täglichen ein- oder zweimaligen Wasserwechsel und für die Zubringung von Nahrung.

Als bemerkenswert soll noch der fehlende Wasserwechsel erwähnt werden, wenn auch bei der stundenlangen Beobachtung in flachen Glasschälchen, woraus man leicht schliessen kann, dass das abgestandene Wasser auf ihre Lebensfähigkeit kaum Einfluss $z \mathrm{u}$ haben scheint. Jedes Tier wurde isoliert in einem abgeteilten Raum des Aquariums gehalten, um seine Eigenart studieren zu können. Die Ernährung und Temperatur der einzelnen Aquarien blieben unbeachtet. Sie hielten sich auf diese Weise ganz vorzüglich und waren dabei den Untersuchungen leicht zugänglich, ohne dass sie erst in andere Gefässe gelegt zu werden brauchten; ausschliesslich für die Schwimmversuche wurden natürlich grössere Aquarien verwendet.

\section{VERSUCHE}

Die Züchtung wurde vom Anfang Mai bis zum letzten Drittel des Juli ausgeführt, denn diese Zeit, ist besonders geeignet für die Züchtung, da sie in ganz enormer Anzahl dort ihr parasitisches Leben führen müssen, und man erbeutet (Mai, Juli) fast ausgewachsene Larven, während man die jüngeren Stadien nur selten zu Gesicht bekommt.

Lebensdauer im Aquarium: Die Häutung konnte ich mehrmals beobachten, und zwar fand ich häufig frisch abgeworfene Hautreste im Aquarium. Die Häutungszahl der Larven ist uns nicht bekannt, aber die völlig ausgewachsenen Larven werden nach der nächsten Häutung $\mathrm{zu}$ geschlechtsreifen Tieren und

$\because$ Als Hauptziel meines Experimentes in dieser Hinsicht nahm ich mir die Lebensdauer der quer durchgesshnittenen Tiere vor. Es gelang mir die beiden Teile 10 Wochen lang am Leben zu erhaiten, länger also als Ditriks, auch die nächste Häutung konnte ich beobachten, aber die Regeneration nicht. 
niemals habe ich an fertigen Nymphonellen einen Hautwechsel beobachtet. Das Tier bleibt elf Wochen lang am Leben in seiner Schale. Die Frage, woher das Tier die Ernährung nimmt, wird vielleicht mit der im folgenden mitgeteilten Tatsache erklärt. Als Nahrung kommt nichts in Betracht, soweit ich es durch Versuche in Aquarien feststellen konnte. Wenn die Tiere aus den Muscheln herausgenommen werden, kann man den Darminhalt, in stets hin und her gehender Bewegung, infolge der peristaltisch arbeitenden Ringmuskeln beobachten; daher ist es leicht zu verstehen, dass die Tiere ziemlich lange Zeit in Aquarien ohne weitere Speisezufuhr leben können. Die Nahrung in Aquarien ist fast ausschliesslich der Darminhalt, denn es findet keine Nahrungsaufnahme statt. Je älter das Tier wird, desto mehr nimmt der Darminhalt $\mathrm{ab}$, und bei der Beobachtung im durchfallenden Licht lässt sich der Blutkreislauf sehr leicht verfolgen. Nun kann man das erwachsene Weibchen leicht mit dem blossen Auge von dem Männchen durch die hellweisse Farbe unterscheiden.

Fortpflanzung: Man kann die völlig geschlechtsreifen Nymphonellen durch Züchtung leicht bekommen, aber die Fortpflanzungsweise konnte leider nicht beobachtet werden. Einmal hatte ich ein Männchen und Weibchen zusammengetan, in der Hoffnung, Paarung oder Eierlegen wahrnehmen zu können. Aber weder Paarung noch Eiablage habe ich gesehen, und durch das Eingehen eines der Tiere im letzten Drittel des Juli, muss ich mich mit einem Hinweise auf diese Frage begnügen.

Ferner bemerkt man eine enorme Anzahl von Lebewesen, die sich auf dem Körper von Pantopoden aufsitztend finden. Ausserdem ist es bekannt, dass die Männchen die Eipäckchen mit sich tragen (OHSHIMA, 1935).

Entwicklung: Nun sehen wir uns die Entwicklung der erwachsenen Larven zu geschlechtsreifen Tieren etwas näher an. Im Anfang des letzten Häutungsverlaufes spalten sich das erste Glied der Palpen in 2 und das neunte Glied in eine Geissel mit zahlreichen Gliedern auf, wie die letzten 2 Glieder des ersten Beinpaares; hier sind Tarsus 1 und 2 durch mehr oder weniger deutliche Septen in eine grosse Anzahl von Gliedern untergeteilt; und gleichzeitig erscheinen die Geschlechtsöffnungen und Stacheln der Gangbeine. Je distaler die Septen beim Beinpaare werden und je älter das Tier wird, desto deutlicher werden sie. Das neunte 
Glied des Palpen, auch die letzten 2 Glieder des ersten Beinpaares, fangen an, sich zu teilen, indem sich das cinzelne Glied weiter in 2 Glieder teilt, jedes wieder in 2 Glieder u.s.w., sodass das Resultat des Aufspaltens eine Geissel bildet. Die grösste von mir wahrge-

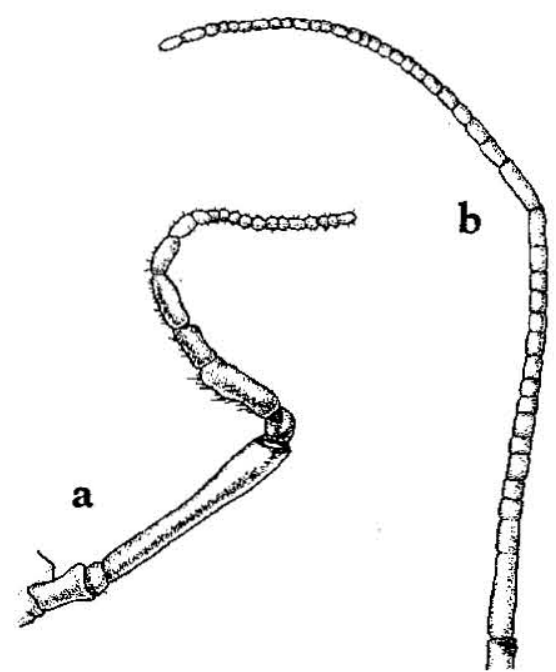

Fig. 1.-Nymphonella tapetis; geschlechtsreifes Tier. a, linker Palpus von Männ. chen, $\times 16 . \quad b$, letzte 2 Glieder von ersten rechten Gangbein des Weibchen, $\times 18$. und später berichtet er (1935): „But if so, what the strangely modified palpi and first pair of walking legs in the female are of use for?", aber die charakteristische Differenzierung von Extremitäten scheint eine phylogenetische, mehr also biologische Bedeutungen zu haben, wenn man die Palaeopantopoden in Betracht zieht.

Unterstützung für die Palaeopantopoden: Unsere Kenntnisse über die fossilen Pantopoden sind noch unvollständig und lücken-

1) Es freut mich, in Hinblick auf die Wichtigkeit dieser Beobachtung, dass die Diagnose der Gattung von Nymphonella von Oısıma nächstens geändert wird.

2) Die Erbeutung der parasitierenden, reifen Tiere gelang mir wiedrholt; $d . h$.

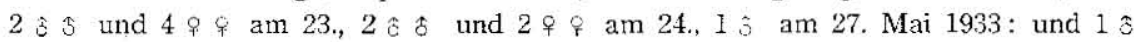
und $1 \%$ am 7. Juni 1936. In den letzten Jahren wurde klar gelegt, wo die Nymphonellen ihre Freileben betreiben. OHshis erbeutete Anfang 1933 weibliche freilebende Individuen im Sand bei Najima(Fukuoka), spater 1935 männliche Individuen bei Amakusa und der Halbinsel Shimabara durch Dredgzug. 
haft, daher herrschen über ihre Beziehungen zu Pantopoden noch verschiedene Ansichten. Als CALMAN und GoRDON (1933) über Palaeopantopus von Brolli schrieben, war ihnen die neue Veröffentlichung von OHsHima (1933) noch nicht zu Gesicht gekommen, wonach man die Palpen mit mehr als 17 Gliedern und die Beine mit mehr als 20 Gliedern von Pantopoden entdeckt hat. Es freut mich, in Hinblick auf die Wichtigkeit dieser Entdekkungen, dass man den wichtigsten dritten Einspruch von CALMAN und GorDoN „3. While three long segments of the leg would correspond to the femur and first and second tibiae of the Pycnogonid, the three short coxal segments of the legs are very obscure, and, what is perhaps the most important character of all, the terminal part of the leg is almost certainly divided into not less than five short articulations " gegenüber BRoLLI, wegnehmen kann, der Palaeopantopus als eine Pantopode berichtet.

Ausschlüpfen der erwachsenen Larve aus ihrem Wirte: Die reifen Tiere betreiben das Freileben (OHshima, 1933), aber die Frage bleibt offen, wie die Tiere denn eigentlich aus ihren Wirten (Muscheln) herausschlüpfen. Obwohl ich, wie OHSHIMA (1927a, 1933) sagt: "It seems highly probable that the parasites leave the bivalve shell after killing it, to become mature and mate", eine Vorstellung von diesen Teilen bekommen habe, war mein Eindruck doch einigermassen anders. Aber die sonstigen Vermutungen von OHSHIMA stimmen meinen überein. Ein wenig später ist er (1935) nicht abgeneigt anzunehmen, dass das Ausschlüpfen geradezu ,aus lebender

Tabelle 1

\begin{tabular}{|c|c|c|c|c|c|c|c|c|c|c|c|}
\hline & $\begin{array}{l}\text { Mai } \\
15\end{array}$ & 20 & 24 & 29 & $\underset{6}{J}$ & 8 & 10 & 11 & 27 & 28 & Summe \\
\hline $\begin{array}{l}\text { Gesamtzahl der lebhaften } \\
\text { Muscheln. }\end{array}$ & 46 & 46 & 46 & 46 & 46 & 46 & 46 & 44 & 44 & 34 & 34 \\
\hline $\begin{array}{l}\text { Gesamtzahl der aus seinen } \\
\text { Wirte herausgekommenen } \\
\text { Nymphonellen. }\end{array}$ & $2(\mathrm{~L})$ & $2(\hat{\mathrm{B}})$ & $\begin{array}{l}2(L) \\
2(6)\end{array}$ & $\frac{2(\mathrm{~L})}{2(\cong)}$ & $2(f)$ & $\begin{array}{l}4\left(\begin{array}{l}4 \\
2(\hat{0})\end{array}\right.\end{array}$ & 0 & 0 & 0 & 0 & $\begin{array}{l}6(\mathrm{~L}) \\
6(\hat{o}) \\
8\left(\begin{array}{c}0 \\
0\end{array}\right)\end{array}$ \\
\hline $\begin{array}{l}\text { Gesamtzahl der schwachen } \\
\text { Muscheln. }\end{array}$ & 0 & 0 & 0 & 0 & 0 & 0 & 2 & 0 & 10 & 0 & - \\
\hline $\begin{array}{l}\text { Gesamtzahl der gestorbenen } \\
\text { Muscheln }\end{array}$ & 0 & 0 & 0 & 0 & 0 & 0 & 0 & 2 & 0 & 10 & 12 \\
\hline
\end{tabular}


Keizô ARITA

Tabelle 2

\begin{tabular}{|c|c|c|c|c|c|c|c|c|}
\hline & $\begin{array}{c}\text { Mai } \\
24\end{array}$ & 25 & 27 & 30 & $\underset{1}{J}$ & 10 & 11 & Sumne \\
\hline $\begin{array}{l}\text { Gesamtzahl der lebhaften } \\
\text { Muszheln. }\end{array}$ & 47 & 47 & 47 & 47 & 43 & 43 & 36 & 34 \\
\hline $\begin{array}{l}\text { Gesamtzahl der aus seinen } \\
\text { Wirte herausgekommenen } \\
\text { Nymphonellen. }\end{array}$ & $1\left\langle L_{i}\right\rangle$ & $\begin{array}{l}1(8) \\
1(3)\end{array}$ & $2(\pi)$ & 0 & 0 & 0 & 0 & $\begin{array}{l}1(\mathrm{~L}) \\
1(\hat{\Omega}) \\
3(\underline{\Xi})\end{array}$ \\
\hline $\begin{array}{l}\text { Gesamtzahl der schwachen } \\
\text { Muscheln. }\end{array}$ & 0 & 0 & 0 & 4 & 0 & 7 & 0 & - \\
\hline $\begin{array}{l}\text { Gesamtzahl der gestorbenen } \\
\text { Mussheln. }\end{array}$ & 0 & 0 & 0 & 0 & 4 & 0 & 7 & 11 \\
\hline
\end{tabular}

Muschel" zu vermuten, was die Differenzierung der Extremitäten zu Hilfe zu ziehen. Die Richtigkeit dieser Vermutung wird durch die nachstehenden Tabellen tatsächlich erwiesen (Tabęlle 1 und 2).

Aus dem mitgeteilten Ergebnisse der künstlichen Züchtung der Muscheln kann indessen, wenn man will, auch ein Schluss gezogen werden. Man kann dann das freilebende Tier im Aquarium beobachten, aber dabei keine abgestorbene oder schwache Muscheln, trotzdem jedesmal, wenn das Tier entdeckt wird, die Muscheln besichtigt wurden. Folglich kann man der Ansicht sein, dass die Tiere aus den lebenden Muscheln herauskommen. Es muss hier auch auf die Tatsache aufmerksam gemacht werden, dass die Schalen der Muscheln nicht immer fest schliessen. Gleichzeitig besteht auch die Möglichkeit, dass die Tiere erst durch das Eingehen der Muscheln zum Freileben übergehen, wie dieses zuerst von OHshima angegeben wurde, besonders wenn die Parasiten in einem Wirte in der Mehrzahl vorhanden sind. Die Tatsache, dass sich das Tier, welches aus seinem Wirte kommt, immer auf dem Sande des Aquariums befindet, lässt darauf schliessen, dass das Tier aus lebenden Muscheln kommt. Anfangs nimmt das Tier die Ruhestellung ein, aber nach wenigen Tagen beginnt seine Bewegung. Der Schmarotzerübergang auf dem Wirte und die Kopulation interessieren uns sehr, weil es sich um Fragen handelt, die man bis jetzt noch nicht beantworten konnte. Aber es wird später die Gelegenheit zur Ausfüllung dieser Lücken gegeben sein.

Defäkation: Einmal hatte ich Gelegenheit, die Defäkation unter 
dem Mikroskop zu verfolgen, und will deshalb eine kurze Schilderung davon nicht unterlassen. Die fertig verdauten Nahrungsklümpchen, die nach und nach Kugelgestalt annehmen, sammeln sich allmählich im Enddarm an. Die mit Kotballen erfüllte Darmflüssigkeit tritt in das Abdomen ein; dann erfolgt ein mässig fester Verschluss, sodass bei der darauf folgenden Kontraktion der Ringmuskulatur die Flüssigkeit wieder in den Darm entweichen kann, während die festen Teile zurückbleiben. Nach mehrmaliger Wiederholung desselben Vorganges wird der Enddarm vollgepfropft; der Verschluss gegen den Rumpfdarm bleibt nun zu, während sich nach einigen einleitenden Muskelzuckungen der Anus plötzlich öffnet und die Kotkügelchen zu einer Säule vereinigt, heraustreten.

Die erste ausführliche Beschreibung vom Zweck der Afteröffnung finden wir bei DoHRN (1881). DoHRN stellte s. Zt. Beobachtungen über den eigentlichen Zweck der Afteröffnung an und meint, sie diene zu einer Art Darmatmung, da er trotz tausendfacher Beobachtungen lebender Pantopoden nie den Austritt geformter Bestandteile aus dem After gesehen habe. Das meint LoMAN (1907), weil DoHRN die lebenden Pantopoden unter dem Deckglas untersuchte, die gegen Druck sehr empfindlich sind und die Verdauung und andere Lebenserscheinungen dadurch alsbald nachliessen. Vielleicht hatte auch DoHRN seine Objekte nicht genügend ernährt. Ein wenig später ist bei. Beobachtungen des Afters Helfer (190G) aber zu dem Schluss gekommen: „Eine bemerkenswerte Eigentümlichkeit bei Pantopoden ist das Fehlen jeder Fäkalmasse, eine Ausscheidung aus dem Darmkanal erfolgt niemals aus dem After, nur aus dem Mund, sodass ersterer überflüssig erscheint, trotzdem er bezüglich Muskulatur u.s.w. vollständig ausgebildet ist." Aber unter dem Deckglas beobachtete ich die Ausscheidung von Fäkalmasse beim Tier, das als Larve absichtlich aus seiner Wirte herausgenommen wurde. Der Einfuss des Druckes ist nicht immer so stark, dass die Verdauung und andere Lebenserscheinungen nachlassen, es ist auszunehmen, dass im Gegenteil die Lebenserscheinungen bei Nymphonella gefördert werden.

Bewegung: Von der Verschiedenheit und der Eigentümlichkeit der Bewegungen kann ich über das Schwimmen, Schreiten, besonders Tauchen (Eingraben in Sand) und so weiter berichten. Auf den ersten Blick findet man auch hier drei von CoLE für 
Pallene festgestellte Formen wieder: Schwimmen, Schreiten und Kombination von beiden, die wohl am besten als "Halbschwimmen " bezeichnet wird. Vortreffliche Beschreibungen und Abbildungen darüber findet man in dem Meisterwerke von Pritr. Die Beschreibung (1909) lautet, dass der hauptsächliche Unterschied. zwischen Schwimmen, Halbschwimmen und Schreiten in der Stärke und Geschwindigkeit der Muskelkontraktion liegt. Bei genauerer Betrachtung aber ergibt sich, dass diese Verhältnisse etwas verwickelt liegen.

Wirft man Nymphonellen aus ihrer Wirten, in das Aquarium, so nehmen sie stets die gleiche Gestalt an, schlagen ihre Beine über der Bauchseite zusammen, die sie als Parasiten in den Muscheln innehalten (Fig. 2), sodass sie wie eine Kugel aussehen, und sinken rasch zu Boden, aber binnen kurzem ergreifen sie sich

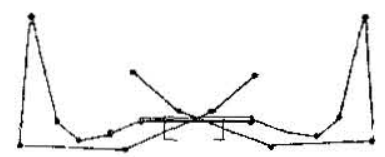

Fig. 2.-Nymphonclla tapetis ; Schema der parasitischen Stellung: von der Vorderseite gesehen. nach langem, planlosem Treiben gegenseitig mit ihren Gliedmassen, einschliesslich Scheren, und bilden schon nach kurzer Zeit einen unentwirrbaren Knäuel, aus welchem sie sich wieder lösen, um endlich die jenem Stadium eigentümlichen Bewegungen zu beginnen, welche im Folgenden beschrieben werden.

Als ich eine grosse Anzahl von parasitierenden Larven in das Aquarium setzte, waren binnen kurzem alle Tiere zu einem Knäuel vereint, wie von Lomas mitgeteilt wurde. Vielleicht lässt sich der Vorgang einfach so erklären, dass die Tięre auseinander $z u$ kommen suchen und dabei beim Tasten nach einer Stütze von neuem sich an den Beinen ihrer Genossen anklammern. LOMAN schloss daraus, dass Phoxichilidium nicht imstande seien, eine Greifklaue wieder zu öffnen, nachdem sie etwas gepackt haben, bevor nicht die übrigen Füsse gleichfalls seinen Halt gefunden hätten; diese Erscheinung aber sei eine wichtige Anpassung an das Leben in der stets unruhigen Litoralzone, damit die Tiere einen einmal bestiegenen Polypenstock nicht einfach verlassen könnten. Diese Eigentümlichkeit ist bei Nymphonella gleichzeitig eine wichtige Anpassung an das parasitische Leben im Wirte, und diese Annahme wird dadurch noch bewiesen, dass diese Anpassung an freilebenden reifen Tiere nicht vorkommt.

Wenn man die Larven besonders für sich in das Aquarium 
setzt, ist die Bewegung anders. Die B€wegung der jungen Larve ist sehr unregelmässig, schwerfällig, so dass von Schwimmen, Halbschwimmen und Schreiten keine Rede sein kann.

Anfangs bewegen sich die erwachsenen Larven unregelmässig, -schwerfällig, die Extremitäten auf den Rücken legend, aber je länger die Zeit vergeht, desto mehr vermehren sich die auf der Bauchseite liegenden Individuen. Um diese Zeit genügt jedoch die geringste Bewegung in der Nähe oder eine leise Berührung, um die Bewegungen mit Kraft aufzunehmen. Wirft man die erwachsenen Larven in ein grosses Aquarium, wie es von PRELL mitgeteilt wurde, so beginnen sie sofort lebhaft $\mathrm{zu}$ schwimmen, und es daucrt oft sehr lange, bis sie schliesslich den Boden erreichen, bald schlagen sie ihre Beine wie erstarrt über dem Rücken schräg nach vorn zusammen und sinken rasch $\mathrm{zu}$ Boden. Sowie dann ihr Körper die Unterlage berührt, schlagen alle Beine auseinander, und das Tier bewegt sich unregelmässig, langsam davon. Sinkt es mit dem Rücken nach unten herab, so werden oft die Beine über die Bauchseite zusammengelegt, und zwar schwankt die Lage dann in der Regel zwischen den beiden abgebildeten Schemata (Fig. $3 \mathrm{~B}_{1}$ und $\mathrm{B}_{2}$ ). Beim willenlosen Absinken, z. B. nach plötzlichem Eindringen in ein anderes Gefäss, klappen meist die Tiere, am Boden angekommen, die Beine nach oben, etwas nach vorn gerichtet (Fig. 3 A). Die erwachsene Larve ist der beste Schwimmer unter allen Stadien, und es ist ihnen möglich

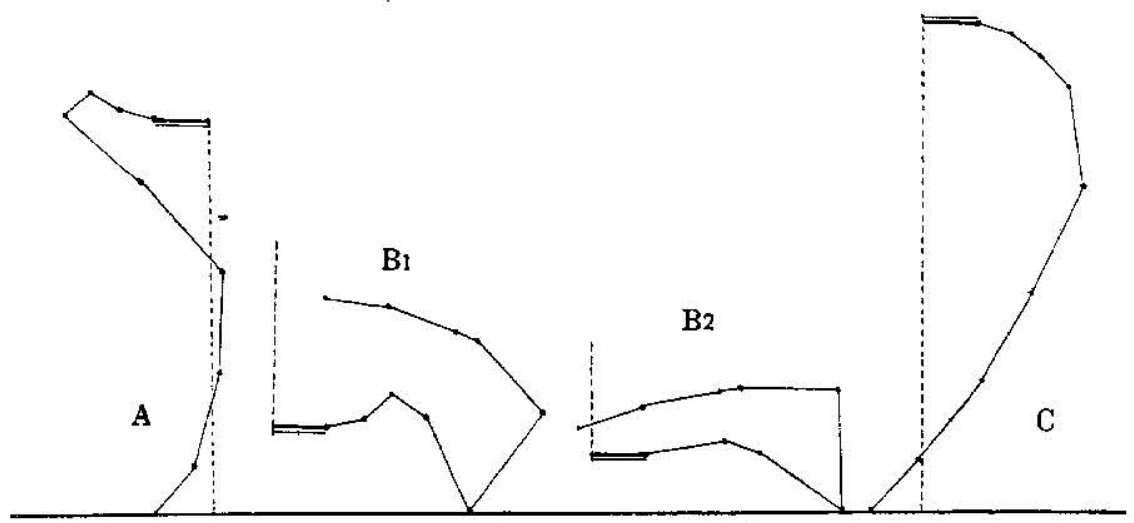

Fig. 3.-Nymphonella tapetis; von der Vorderseite gesehen. A. Schema von Unter lage zu Normallage kehrend. $B_{1}$. und $B_{2}$. Schemata der Ruhestellungen mit dem Rücken nach unten. C. Schema von Normallage zu Lnterlage werdend. 
sich vom Boden zu erheben. Halbschwimmen wurde nicht beobachtet.

Schwimmen und Schreiten sind von COLE und PRELL beobachtet und beschrieben, sodass auf den ersten Blick eine hinzugefügte Bemerkung nicht notwendig erscheint. Aber es ergibt sich bei. genauerer Betrachtung, dass die Verhältnisse für Nymphonella etwas verwickelter liegen. Die Beobachtung Prells, die den Hauptunterschied zwischen dem Schwimmen und dem Schreiten in der Stärke und Geschwindigkeit der Muskelkontraktion findet, passt gänzlich auf Nymphonella, wenn man daraufhin ein Gangbein beobachtet. Da die Schwimmbewegungen ziemlich rasch erfolgen, ist es nicht leicht, die Folge der einzelnen Phasen sicher zu erkennen.

Vom Schwimmen als der lebhaftesten und stärksten Bewegung ausgehend, hat PREIL ein bemerkenswertes ",Schema der Phasenfolge bei einem Ruderschlage von Nymphon " aufgestellt, das hier nicht fehlen soll. Fr betont zwar ausdrücklich, dass dieses Schema nicht bindend sei, vielmehr Schnelligkeit und Ausführung der einzelnen Bewegungen von dem physiologischen Zustand des betreffenden Individuums stark abhängig sind.

Der Schwimmrhythmus von Nymphonella ist aber einigermassen anders. Im allgemeinen vollzieht sich der Verlauf des Schwimmrhythmus' in folgender Weise. Was den Rhythmus der Beinbewegung anbelangt, so ist er weniger klar zu erkennen. Konstant ist allein, dass die gleichen Phasen von hinten nach vorn fortschreiten, wobei nicht die beiderseitigen sich entsprechenden Gangbeine bewegt werden. Vielmehr zeigt sich, dass dic Beinc II und IV derselben Seite in etwa gleicher Lage stehen; und auf der Gegenseite die Beine I und III ziemlich dieselbe Stellung in diesem Augenblick einnehmen.

Aber je mehr die Beine nach vorn stehen, desto mehr verspätet sich die Phase des Rhythmus. Darauf basierend kann man sich leicht eine Kombination zusammenstellen und erhält dabei das auf der Abbildung (Fig. 4) dargestellte Schema, in dem die verstärkte Linie ein schlagendes, die feine ein ausholendes Bein bedeutet. Die Beine sind dabei als einfache Linien gezeichnet. Man kann sogar leicht die einzelne Phase der Gangbeine zu jeder beliebigen Zeit erkennen, indem man die Abbildung (Fig. 4 C) zu Hilfe zieht. Auf der Abbildung (Fig. $4 \mathrm{~A}$ und $\mathrm{C}$ ) habe ich die Schlagbreite eines Beines in vier Phasen zerlegt. 
Dass die Konstanz des Neigungswinkels von grösster Bedeutung für das Tier ist, geht aus der Richtung des Schwimmens hervor. Es ist nämlich die Kraft des Ruderschlages fast ausschliesslich ein

A 1

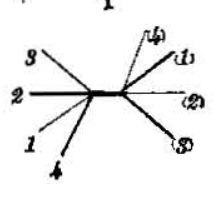

B 1

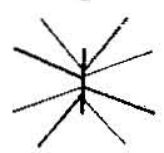

2

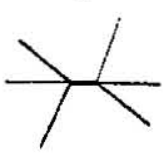

2

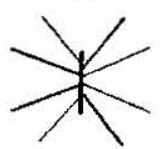

3

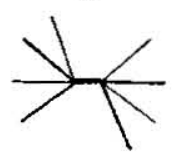

3

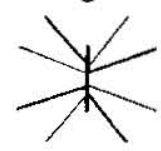

4
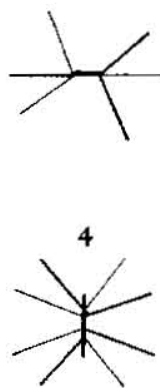

5

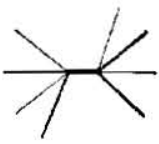

5

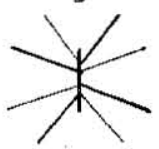

C

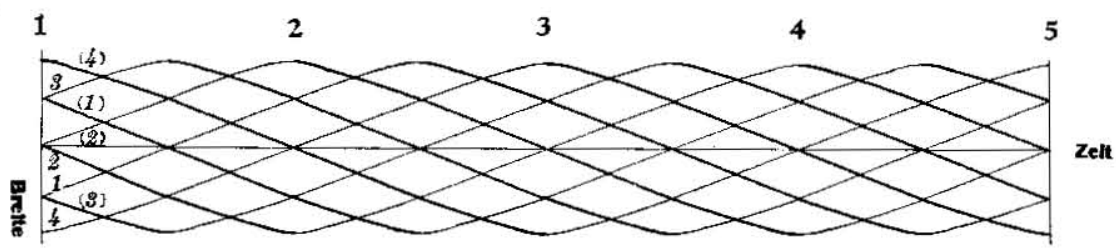

Fig. 4.-Nymphonella tapetis; Schemata der Phasenfolge und des Rhythmus beim Schwimmen. Folge von links zu rechts; und $A_{1}, B_{1}$ und $C_{1}$ stehen in gleicher Stellung. A. von der Vorderseite gesehen. B. von der Oberseite gesehen. C. Verlauf der Phasenfolge.

Heben des Tieres dorsalwärts. Zur horizontalen Fortbewegung muss das Tier seinen Rücken schräg stellen, sodass die vertikale Komponente seiner Geschwindigkeit die Gravitation aufhebt und die horizontale es vorwärts treibt. Könnte nun die Neigungsebene nicht eingehalten werden, so wäre es dem Tiere unmöglich, eine bestimmte Richtung zu wahren; das ,zielbewusste " Schwimmen wäre also umgewandelt in ein mehr oder weniger hilfloses Treiben.

Die Schreitbewegung ist nicht deutlich, und kann nur an einer erwachsener Larve mit vieler Mühe wahrgenommen werden. Anfangs ist es ziemlich schwierig zwischen der unregelmässigen Bewegung und dem Schreiten zu unterscheiden. Merkwürdig im Vergleich mit den anderen Pantopoden ist ihre ausgesprochene Differenzierung vom 1. Gangbeinpaar. Die Rolle des 1. Gangbeinpaares der erwachsenen Larve weicht, wie gesagt, von den übrigen Beinpaaren durch das charakteristische Gliedern der 1. und 2. 
Tarsen $a b$, sodass das Schreiten durch die übrigen 3 Beinpaare ausgeführt wird. Welche Rolle die differenzierten Extremitäten im Leben dieser Tiere spielen, kann ich nicht entscheiden.

Die Schreitbewegung weicht, wie gesagt, in ihrem Rhythmus sehr von dem des Schwimmens ab, aber das Schreiten ist in seinen Einzelheiten nichts anderes, als eine verlangsamte Schwimmbewegung. Der Körper schwebt beim Gehen ziemlich hoch über dem Boden; bei den einzelnen Schritten pflegt er etwas emporzuwippen. Die Gelenke der Beine erleiden alle eine durchschnittlich geringere Beugung als beim freien Schwimmen. Die Beinbewegung erfolgt rhythmisch, und der Verlauf des Schreitrhythmus ist ungefähr folgender: Die Extremitäten $\mathrm{V}$ und VII derselben Seite stehen in gleicher Phase; die beiden Körperhälften differieren dabei in ihrer Tätigkeit so miteinander, dass auf der Gegenseite die Extremität VI sich ebenfalls in derselben Lage befindet. Darauf basierend kann man sich leicht eine Kombination zeichnen und erhält dabei

Fig. 5.-Nymphonella tapotis; Schema des Schreitenrhythmus: von der Dorsalseite gesehen. das auf der Abbildung (Fig. 5) dargestellte Schema, in dem die verstärkte Linie ein schlagendes, die feine ein ausholendes Bein bedeutet.

Merkwürdig sind die zierlichen Stacheln, welche den vier distalen Gliedern der Eiträger an der Innenseite aufsitzen. DoHRN, der sie als eichenblattförmige Anhänge bezeichnet, betrachtet sie als Hilfsorgane zum Tragen der Eier. Diese konnte ich jedoch bei Nymphonella leider nicht beobachten. Einer ganz besonderen Bewegungsmöglichkeit der Eiträger sei auch gedacht, an deren vier distalen Gliedern sich blattartige Anhänge befinden, die nach Art „Ringbürste“ (PRELl, 1910) wirken. Dieses fand ich auch durch meine Beobachtung bestätigt. Ein in Ruhe befindliches Tier pflegt sich unermüdlich zu putzen, wobei der Körper dabei der Tätigkeit der Bürste zugänglich ist, obschon der Rumpf in dieser Beziehung am wenigsten begünstigt ist. So wird auch das Abdomen von eventuell anhaftenden Kotresten gesäubert. Auch reinigen sich untereinander die Eiträger.

Man erinnert sich hierbei der von Loman (1905) bei Decolopoda und Colossendeis angestellten Betrachtungen, wobei auch er schon meinte, dass die Eiträger nicht ausschliesslich der Funktion des Eitragens, sondern auch $z u$ anderen Zwecken, für die genannten 
Genera z.B. als Ernährungsorgan, dienen, indem sie dort beim Absuchen des Seebodens infolge ihrer Länge behilflich sein können, und besonders die vier Endglieder zu einem wirksamen Greiforgan wurden.

Erwähnt sei noch die neuere Beobachtung über die besondere Bewegungsmöglichkeit der Eiträger, die nach Art eines „Tauchsorganes " wirken. Von dieser besonderen Bewegungsmöglichkeit ist an anderer Stelle ausführlich die Rede (s. S. 286: Fig. 6).

Wiederholt kann man auf dem Rücken liegende Larven sehen. Auf weiche Weise sie zu dieser Lage kommen, wurde leider nicht beobachtet. Bald nähert das Tier die Spitzten sämtlicher Beine nach der Bauchseite gegeneinander, sodass sie wie ein Quirl aussehen (Fig. 3 A), bis der Körper das Übergeweicht bekommt, umkippt, und auf die Rückenseite fällt; manchmal fällt es auch während des Schwimmens auf die Rückenseite.

Zuletzt wollen wir zur Beschreibung von ausgewachsenen Nymphonella übergehen. Die Bewegungen beim reifen Tier sind ziemlich anders. Das sieht man schon daraus, dass sie, einmal plötzlich von Muscheln losgelöst und ins Aquarium getan, eine Zeitlang hilflos eine Kugelform (Fig. 2) einnehmen; aber binnen kurzem fangen sie an, sich $z u$ bewegen. Fällt ein reifes Tier frei durchs Wasser, so pflegt es sich folgendermassen zu bewegen: Einmal durcheilt es schwimmend mit grosser Hast seinen Behälter, während es ein anderes Mal seine Beine über dem Rücken schräg nach vorn zusammenschlägt, und sinkt rasch zu Boden. Sinkt es einmal zu Boden, geschieht das Schreiten nicht. Geschieht es, so nur eine sehr kurze Strecke weit, und dann beginnt das Tauchen.

Als sehr merkwürdig soll das von mir ausgesprochene „Tauchen" erwähnt werden, welches leider bisher bei den Pantopoden noch nicht beobachtet wurde, soweit ich weiss. Das Tauchen kann man nur bei reifen Tieren beobachten, und vielleicht wird man in der Lebensweise seine Begründung finden. Bei einem Leben auf unebenem Untergrund, vor allem in der Küstenzone, wo ausserdem durch Brandung und Strom die Pantopoden lebhaft bewegt werden, ist eine besondere Sicherheit nützlich; hierfür dient bekanntlich das Eingraben in Sand. Die Abbildung soll, wie beim Schwimmen, an Hand eines konkreten Falles den allgemeinen Charakter des Tauchens schildern. Auf der Abbildung (Fig. 6) 
habe ich die Bewegung in 9 Phasen zerlegt. Die Glieder sind dabei als einfache Linien gezeichnet, da eine vollständige Ausführung nur der Klarheit Abbruch tun würde; die punktierte Basis deutet die Bodenebene an.
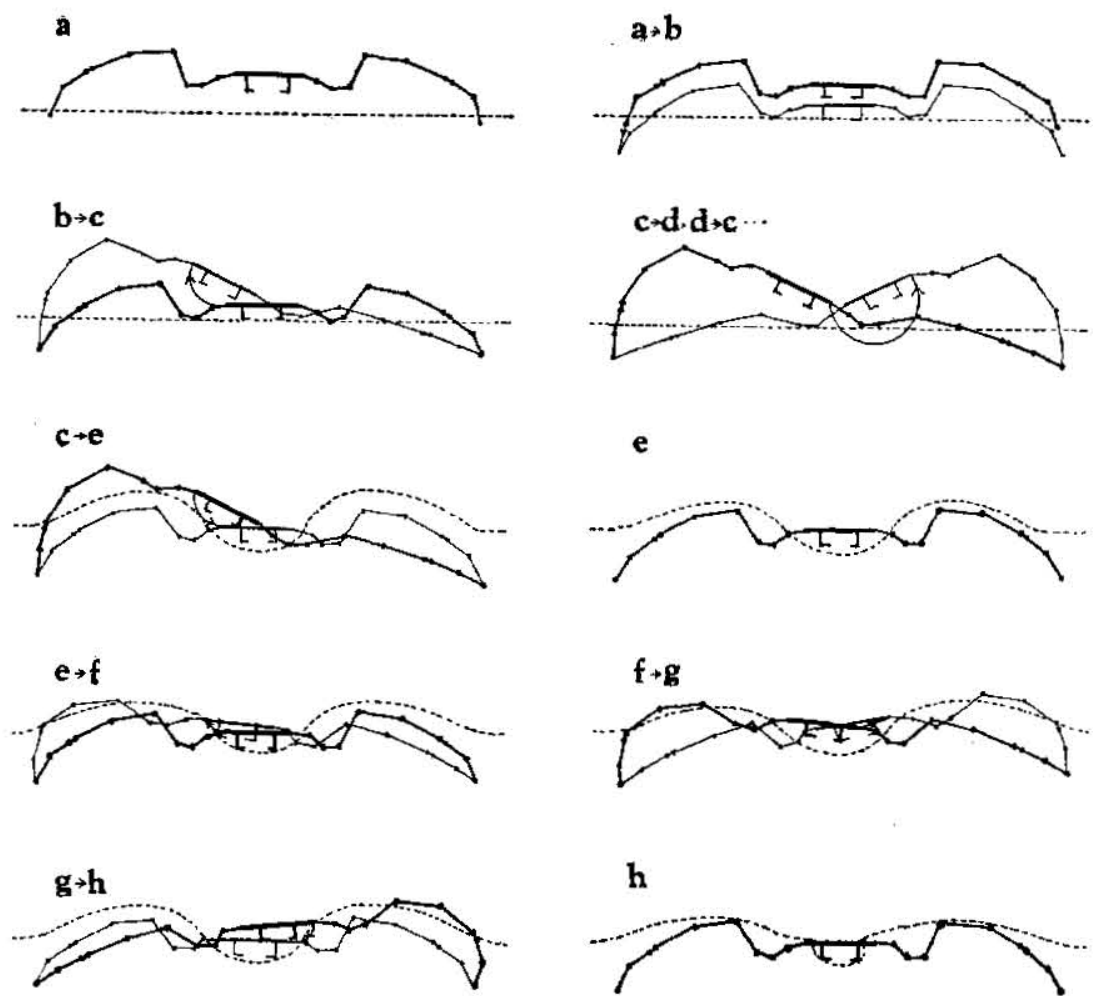

Fig. 6.-Nymphonella tapetis; Schemata der Phasenfolge beim Tauchen: von der Vorderseite gesehen.

Der Verlauf des Tauchens ist ungefähr folgender. Zu Anfang geschieht das Einschlagen der Klaue im Boden, welches durch die 2., 3. und 4. Gangbeine ausgeführt wird (Fig. 7), bei dem die Beine 2 und 4 der entgegengesetzten Seite in gleicher Phase stehen; die anderen 2 Partner dabei in ihrer Tätigkeit so miteinander ruhen, dass die entgegengesetzten Beine 2 und 4 in Ruhestellung sind und das 3. Beinpaar in gleicher Phase unabhängig mit dem 2. und 4. Beinpaare stehen. Hin und wieder konnte man beobachten, dass bald das 2. linke und 3. rechte und bald das 4 . linke und 3. rechte Bein oder umgekehrt in gleicher Phase stehen. 
Das Einschlagen wird bis $\mathrm{zu}$ jedem 2. Tarsen (Fig. 6 b) durchgeführt, folglich wird das Gerüst sicher. Dann hebt es zu tauchen an mit dem 3. Basalglied, Femur und Eiträger, die beiden Körperhälften differieren dabei in ihrer Tätigkeit gerade entgegengesetzt miteinander. Nach 3-5 Malen desselben Vorganges $(\mathrm{c} \rightarrow \mathrm{d}, \mathrm{d} \rightarrow \mathrm{c} \ldots$ ) wird das Gerüst sicher, und dann beginnt das Tauchen von neuem. Kommt die Oberfläche des Rückens des Tieres gleich mit derselben des Bodens (e) hört cs mit Tauchen auf. Zuletzt erschüttert sich der Körper $(\mathrm{e} \rightarrow \mathrm{f}, \mathrm{f} \rightarrow \mathrm{g} \ldots)$, dann die Sandmasse, die an den beiden Seiten anhäuft, zerbröckelt über dem Körper (h). Es

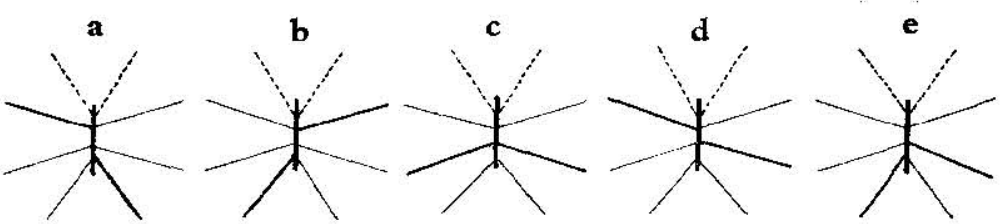

Fig. 7.-Nymphonella tapetis; Schemata des Einschlagenrhythmus des Beines beim Tauchen: von der Oberseite gesehen.

ist dann in der Tat sehr schwer, im Sand tauchende Nymphonellen auf den ersten Blick zu erkennen. Zuweilen konnte ich beobachten, dass das Einbetten auf der Mitte des Weges (bis e) aufhört, aber das ist ein anormaler Fall.

\section{ZUSAMMENFASSUNG}

1. Die Nymphonellen sind leicht elf Wochen lang im Aquarium zu halten mit sich selbstreinigendem Wasser und Sand.

2. Die Bewegungen der jungen Larven erfolgen unregelmässig, schwerfällig auf dem Rücken liegend, dieselben der erwachsenen Larven erfolgen hauptsächlich durch Schwimmen und am wenigsten durch Schreiten, und dieselben der gereiften Tiere erfolgen in der Hauptsache durch Tauchen, während Schreiten kaum beobachtet wurde.

3. Nach der letzten Häutung vermehren die Tiere ihre Gliederzahl der Extremitäten II und IV.

4. Die grösste von mir wahrgenommene Anzahl beträgt 22 an Palpe, und in Hinblick auf die Wichtigkeit dieser Beobachtung wird die Diagnose der Gattung von Nymphonella von OHshima geändert. 
5. Das Vermehren der Glieder der Extremitäten vermag den wichtigsten Einspruch von CALMAN und GORDON gegenüber BRolli wegzunehmen, der Palaeopantopoden für eine Pantopode hält.

6. Man kann der Ansicht sein, dass die Tiere aus den lebenden Muscheln herauskommen.

7. Das Schreiten erfolgt durch Extremitäten V, VI und VII.

8. Das Tauchen erfolgt in der Hauptsache mit Hilfe der Gangbeine und Eiträger.

9. Man kann die Defäkation an den Tieren bemerken, die unter dem Deckglas liegen.

10. Bald nähert das Tier die Spitzen sämtlicher Beine nach der Bauchseite gegeneinander, bis der Körper das Übergewicht bekommt, umkippt und auf die Rückenseite fällt; ebenfalls während des Schwimmens auf die Rückenseite fällt.

(Zoologisches Laboratorium, Kaiserliche Kyûshû-Universität.)

\section{ZITIERTE LITERATUR}

Calman, W. T. und I. Gordon. 1933 A Dodecapodous Pycnogonid. Proc. Roy. Soc. London, Vol. 113, p. 107-115.

Cotr, L. J. 1901 Notes on the habits of Pycnogonids. Biol. Bull. Boston, Vol. 2, p. 195-207.

Durk, A. 1881 Die Pantopoden des Golfes von Neapel und der angrenzenden Meeresabschnitte. Fauna u. Flora d. Golfes von Neapel, Bd. 3, S. 1-252.

Helfer, H. 1939 Biologisch-faunistische Beobachtungen an Pantopoden der Nordund Ostsee. Inaug.-Dissert. Kiel., S. 1-48.

Lols, J. 1896 Ueber die Regeneration des Rumpfes bei Pantopoden. Zur Theorie der Regenerationserscheinungen. Arch. Entwicklungsmech., Bd. 2, S. 250-256.

Loman, J. C. C. 1905 (Zitiert von Hiafir, H. und E. Schlottke, 1935, In Bronns Klassen und Ordnungen des Tierreichs, Bd. 5, S. 1-308.)

1907 Biologische Beobachtungen an einem Pantopoden. Tijdschr. Nederland. Dierk. Ver. Leiden (2) Bd. 10, S. 255-284.

Onsmma, H. 1927a Nymphonclla tapetis n. g. n. sp., a Pycnogon parasitic in a Bivalve. Annot. Zool. Japon., Vol. 11, p. 257-263.

- 1927b Piknogono parazite vivanta en bivalvo (Japanisch mit Esperanto résumé). Bult. S:i. Fakult. Terkult., Kjuŝu Imp. Univ., Vol. 2, p. 366-379.

- 1933 The adult of the bivalve-infesting Pycnogonid, Nymphonella tapetis Orsmma. Annot. Zool. Japon., Vol. 14, p. 53-60.

- 1935 A further note on Nymphonella lapotis; the egg-carrying mature male (Eurycydidae, Pantopoda). Annot. Zool. Japon., Vol. 15, p. 95-102.

Prficl, H. 1910 Beitrage zur Kenntnis der Lebensweise einiger Pantopoden. Bergens Mus. Arb. (Naturvid. R.)., Nr. 10, S. 1-30. 